K.C. Freeman

Mount Stromlo and Siding Spring Observatories

Research School of Physical Sciences

The Australian National University

\title{
INTRODUCTION
}

First I will describe briefly some older work on chemical gradients in globular clusters. Then I will discuss some more recent data on the chemical and kinematical properties of 47 Tuc and Omega Cen, that leave us in little doubt about the reality of such chemical gradients.

\section{REVIEW OF OLDER WORK}

Many years ago, Martin (1937) demonstrated that the radial distributions of RR Lyrae stars and red giants in Omega Cen are clearly different: the RR Lyrae stars are less centrally concentrated. This was originally believed to result from dynamical relaxation, because the masses of the RR Lyrae stars are less than those of the red giants. However we now know that the relaxation time at the center of this cluster is significantly longer than the total lifetime on the giant branch and the horizontal branch, so this explanation seems unlikely. Another possibility suggested by this observation is that the cluster has a radial gradient of chemical abundance, because the morphology of the horizontal branch is sensitive to abundance. This suggestion led to much of the recent work on abundance gradients in clusters. We will see later that the direct evidence for a chemical gradient in this cluster is very strong indeed.

In external galaxies, chemical gradients are seen most easily in the form of color gradients. Such color gradients have also been observed in some globular clusters. Gascoigne and Burr (1956) first reported the color gradient in 47 Tuc. This was confirmed by chun and Freeman (1979); they also made photoelectric UBV concentric aperture of several other clusters, which showed that some clusters have color gradients and some do not. They noticed that the clusters with color gradients are those whose central relaxation times are longer than about 1.0 years. This makes sense if the color gradients are set up early in the life of the clusters; the gradients could then survive to 33

J. Goodman and P. Hut (eds.), Dynamic's of Star Clusters, $33 \quad 42$.

(C) 1985 by the IAU. 
the present time only in the clusters with relatively long relaxation times.

In globular clusters, most of the visible light comes from the giants, so the color gradients must be associated with some change in the bright end of the color-magnitude diagram or of the luminosity function. Chun (1976) studied the color-magnitude diagram for 47 Tuc in several radial zones; he showed that the CMD for the giant branch is very similar at all radii. However the bright end of the luminosity function does change with radius. There are more bright giants $\left(\mathrm{M}_{\mathrm{V}}<-1\right)$ per unit luminosity in the inner regions. A similar radial change in the luminosity function has been found in Omega Cen (Freeman, unpublished). Again, these luminosity function changes are clearly not dynamical in origin, because the masses of all the giants are almost the same. Chun was able to show that, for 47 Tuc, the radial changes in the bright end of the luminosity function are sufficient to produce the observed color gradient.

It seems, then, that the color gradients are associated with luminosity function gradients. However the reason for these gradients is not yet understood. Probably, it has to do with chemical gradients (Freeman, 1978; d'Antona et al, 1984) or possibly with radial changes in some other quantity, like stellar rotation (Norris, 1981). I have suggested (Freeman, 1978) that the excess bright giants could be AGB stars; their numbers are sensitive to the distribution of stars on the $\mathrm{HB}$, which in turn could be affected by radial changes in abundance. To get more AGB stars near the tip of the giant branch, towards the cluster center, we would need a redder HB towards the center. From chun's photometry in $47 \mathrm{Tuc}$, it seems that the $\mathrm{HB}$ is indeed redder in the inner parts where the excess of bright giants is found.

There is some direct older evidence for chemical gradients in globular clusters. Firstly, many clusters have $\mathrm{C}$ and $\mathrm{N}$ inhomogeneities which are seen most readily as star to star variations in the strength of the $\mathrm{CN}$ bands in the stellar spectra. The distribution of $\mathrm{CN}$ band strengths is frequently bimodal (Norris and Smith, 1981). One such cluster is 47 Tuc. From a sample of about 140 giants with measured CN band strengths, Norris and Freeman (1979) found that the relative strength of the two $\mathrm{CN}$ modes changes markedly with radius in the cluster. (The CN-strong mode is most prominent near the cluster center). Smith (1979) has also detected a radial CN gradient from integrated spectra of the inner parts of 47 Tuc. In Omega Cen, Norris et al (unpublished) have a sample of about 200 stars with measured CN strengths, all the way from the cluster center out to the tidal radius. This sample shows a spectacular $\mathrm{CN}$ gradient: the inner parts have stars covering a wide range of $\mathrm{CN}$ strengths, while the outer parts have only $\mathrm{CN}$-weak stars (see Figure 4). I will discuss the CN gradients in 47 Tuc and Omega Cen again, later. However, it should be said here that there is still disagreement about whether the $\mathrm{CN}$ spread (and therefore the CN gradients) come from mixing processes within these low mass stars, or from accretion of $\mathrm{CN}-r i c h$ material on to the surfaces of these stars early in the 
life of the cluster, or from $\mathrm{CN}$ inhomogeneities in the gas from which the cluster formed. See Freeman and Norris (1981) for a discussion of this problem.

Secondly, Omega Cen shows star to star inhomogeneities in the heavy element abundances (see Freeman and Norris 1981). Only Omega Cen and M22 (to a lesser degree) have this property. This inhomogeneity is seen very well in the sample of $58 \mathrm{RR}$ Lyrae stars with measured $[\mathrm{Ca} / \mathrm{H}]$ values (Butler et al, 1978). These RR Lyrae stars show a clear radial gradient in $[\mathrm{Ca} / \mathrm{H}]$; again, the inner stars are more Ca-strong (Freeman, 1978; Smith, 1981). The heavy element inhomogeneity in Omega Cen is particularly interesting, because these elements cannot be produced in the low mass stars that we are now observing in this cluster. We do not yet understand the origin of the heavy element inhomogeneity and radial gradient in Omega Cen, but it seems very likely that they were established early in the life of the cluster. Maybe they were produced by selfenrichment at the time of cluster formation, while the massive stars were evolving, or come from the merger of pre-cluster fragments of different abundances (Searle and Zinn, 1978). In Omega Cen, the relaxation time is so long that a radial abundance gradient would readily survive to the present time.

THE RADIAL DISTRIBUTION OF CN IN 47 TUC

Figure 1 shows the radial distribution of $\mathrm{CN}$ strength for Norris and Freeman's (1979) sample of giants in 47 Tuc. This sample extends out from the center of the cluster to about half the tidal radius. The $\mathrm{CN}$ gradient is not immediately obvious, because we see stars with strong and weak $\mathrm{CN}$ over the whole range of radius. However the gradient is seen clearly when we compare the frequency distributions of CN strength at different radii. Generalised histograms of the CN distribution in three radial intervals are shown in Figure 2. The relative population of the two $\mathrm{CN}$ modes changes with radius: the inner parts of the cluster have a much larger proportion of $\mathrm{CN}$-strong stars than do the outer parts.

Da Costa and I have accurate radial velocities for this sample of stars in 47 Tuc (see our paper in this volume). We have examined this velocity data, to see whether the $\mathrm{CN}$ gradient has any associated kinematical effects. For example, the $\mathrm{CN}$-strong stars are more centrally concentrated, so one might expect that their velocity dispersion would be smaller, particularly at larger radii. However, we were unable to find any significant dependence of velocity dispersion on $\mathrm{CN}$, at any radius. The lack of an observable effect probably results from the limited radial range of our sample (few of the stars have $R>R_{t} / 3$ ). We also estimated the mean rotation of the $\mathrm{CN}$-weak and $\mathrm{CN}$-strong subsamples: both rotate, but there is again no significant difference.

In summary, 47 Tuc has a well-defined CN gradient. Whatever produces the two modes in the $\mathrm{CN}$ distribution (whether abundance bimodality or some mixing process) is itself radially varying. 


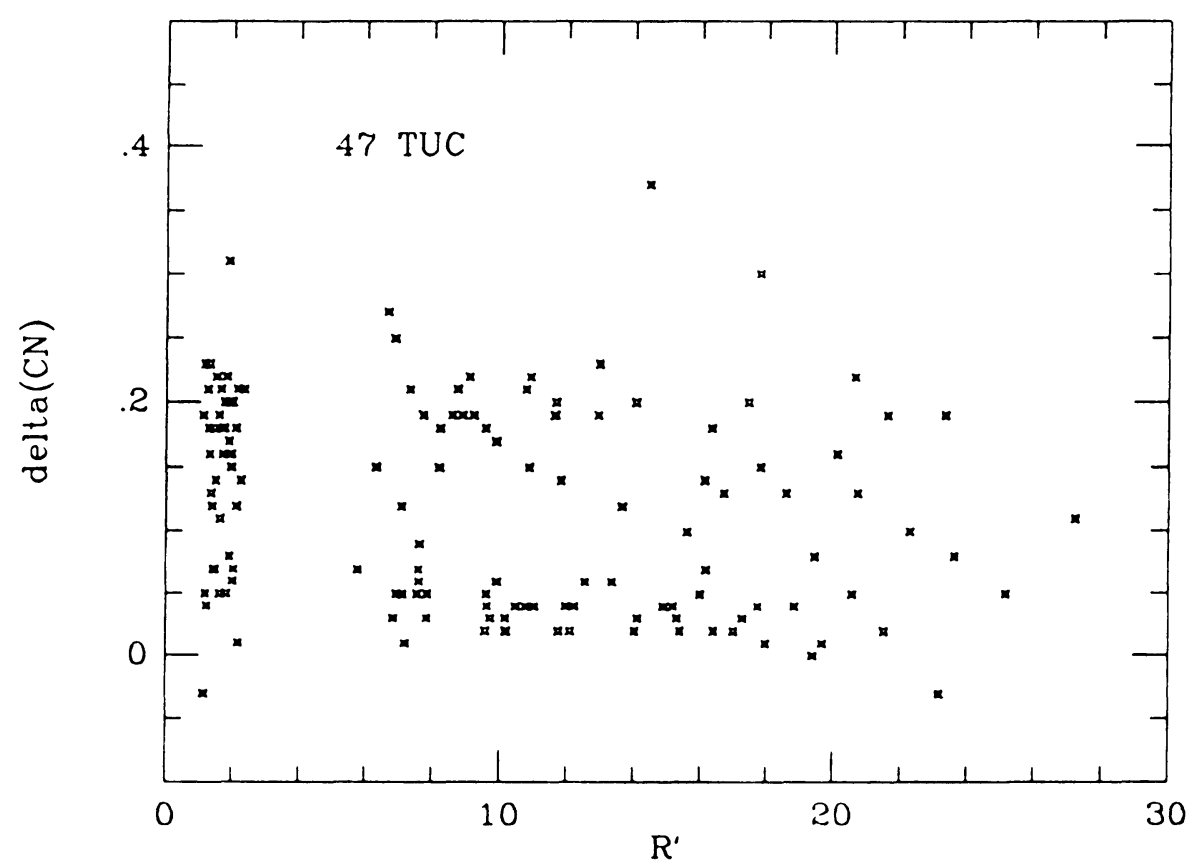

Figure 1. The radial distribution of $\mathrm{CN}$ strength delta(CN) for stars in 47 Tuc. High values of delta (CN) correspond to strong $\mathrm{CN}$ band strengths. Note the wide range of delta $(\mathrm{CN})$ at all radii.

A comment on the persistence of the CN gradient in 47 Tuc: in the inner parts of the cluster $\left(R<2^{\prime} .5\right)$, no gradient is visible (see Figure 3 ). In this region, $R<5 R$, where ${ }_{9}{ }_{C}$ is the core radius, and the relaxation time is less than about $10^{9}$ years. At the next radial zone shown in Figure 2, where $\mathrm{R}>12 \mathrm{R}$ and the relaxation time is longer than the age of the cluster, we see that the $\mathrm{CN}$ gradient has clearly set in. This is all consistent with the $\mathrm{CN}$ gradient having been established early in the cluster's life and now diffusing away through dynamical relaxation: the inner parts of the cluster have already been homogenised by relaxation. It is unfortunate that there are no stars in our sample in the radial zone $5 R<R<12 R$, because in this region we should see the $\mathrm{CN}$ gradient gradually appearing. This would be a nice test of the above picture. We will observe a sample of giants in this region in the coming season.

\section{THE RADIAL CN DISTRIBUTION AND KINEMATICS OF OMEGA CEN}

Omega cen is the chemically inhomogeneous cluster. It also has a very long relaxation time, so any initial chemical gradients would have an excellent chance of surviving to the present time, almost unmodified by 

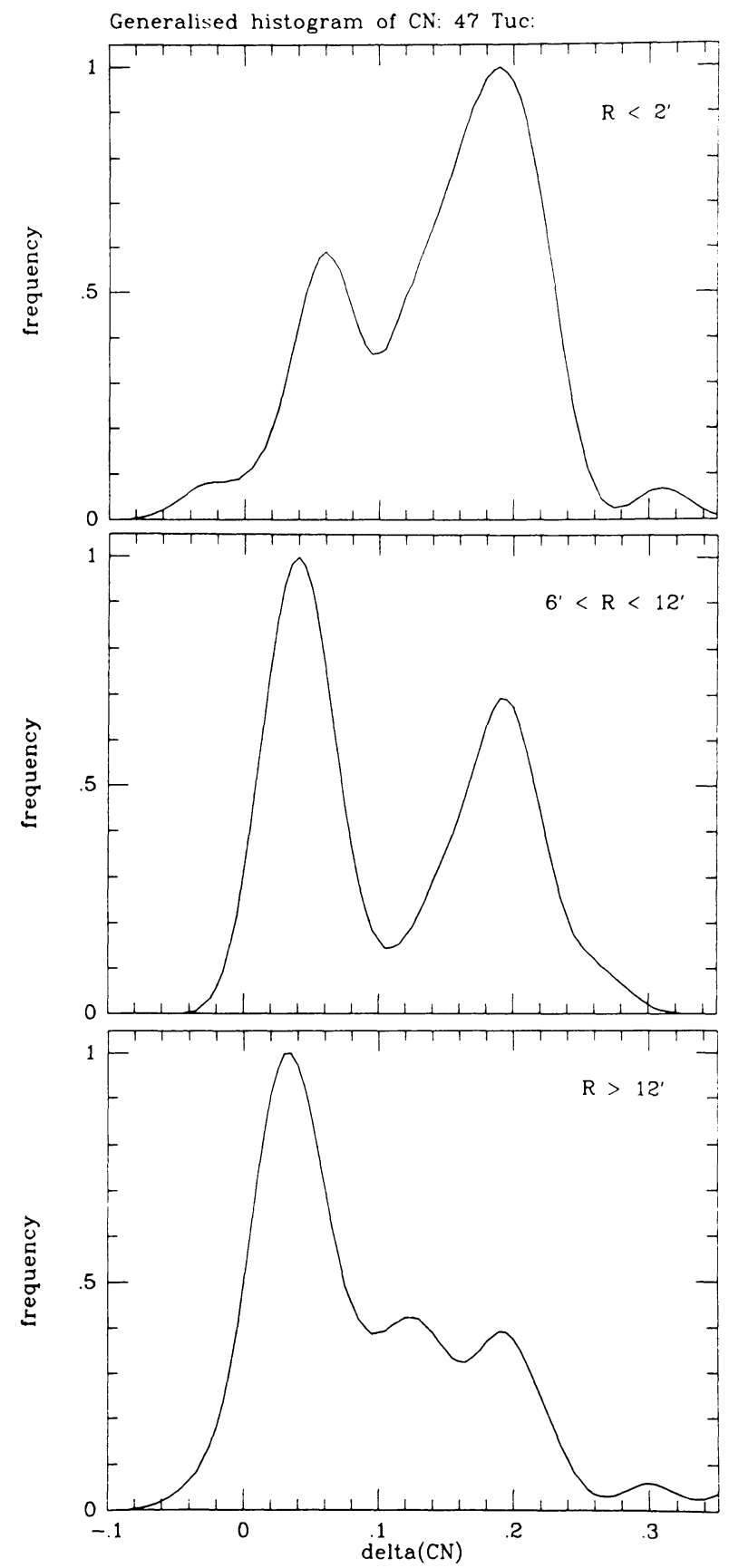

Figure 2. Generalised histograms of $\mathrm{CN}$ strength for stars in three radial zones in 47 Tuc. Note how the relative strength of the two $\mathrm{CN}$ modes changes with radius. 
47 Tuc: inner region

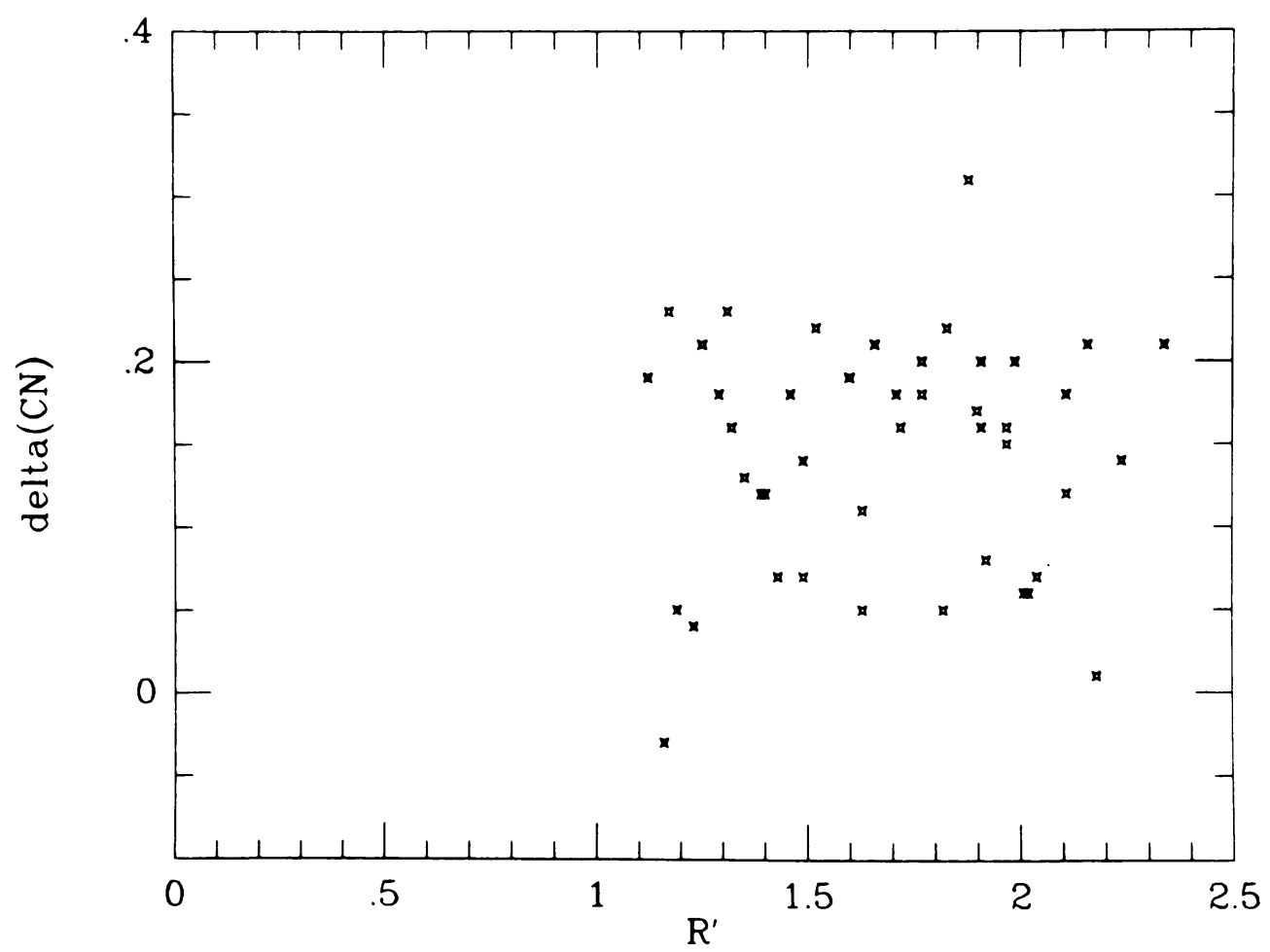

Figure 3. The radial distribution of $\mathrm{CN}$ strength for stars in the inner region of $47 \mathrm{Tuc}$. Note the absence of any $\mathrm{CN}$ gradient in this region.

relaxation effects. Furthermore, Omega cen is a very massive and not very highly concentrated cluster, and it has a large systemic radial velocity: together, these properties make it relatively easy to find member stars in the outermost parts of the cluster. Following a program by Seitzer and Freeman to find such stars, we now have a sample of 115 red giants, with measured $\mathrm{CN}$ strengths and accurate radial velocities, from the cluster center out to the tidal radius.

Figure 4 shows the $\mathrm{CN}$-radius diagram for these stars. The $\mathrm{CN}$ gradient in the outer half of the cluster is unmistakable. The outer parts of the cluster are $\mathrm{CN}$-weak, while the inner parts have stars with a wide range of $\mathrm{CN}$ strengths. Such an obvious $\mathrm{CN}$ gradient should have a kinematical counterpart. This is seen in the velocity dispersionradius relation for stars with weak, intermediate and strong $\mathrm{CN}$, shown in Figure 5. The $\mathrm{CN}$-strong stars are found only in the inner half of the cluster. Near the maximum radius at which these stars are found, 


\section{OMEGA CEN STARS}

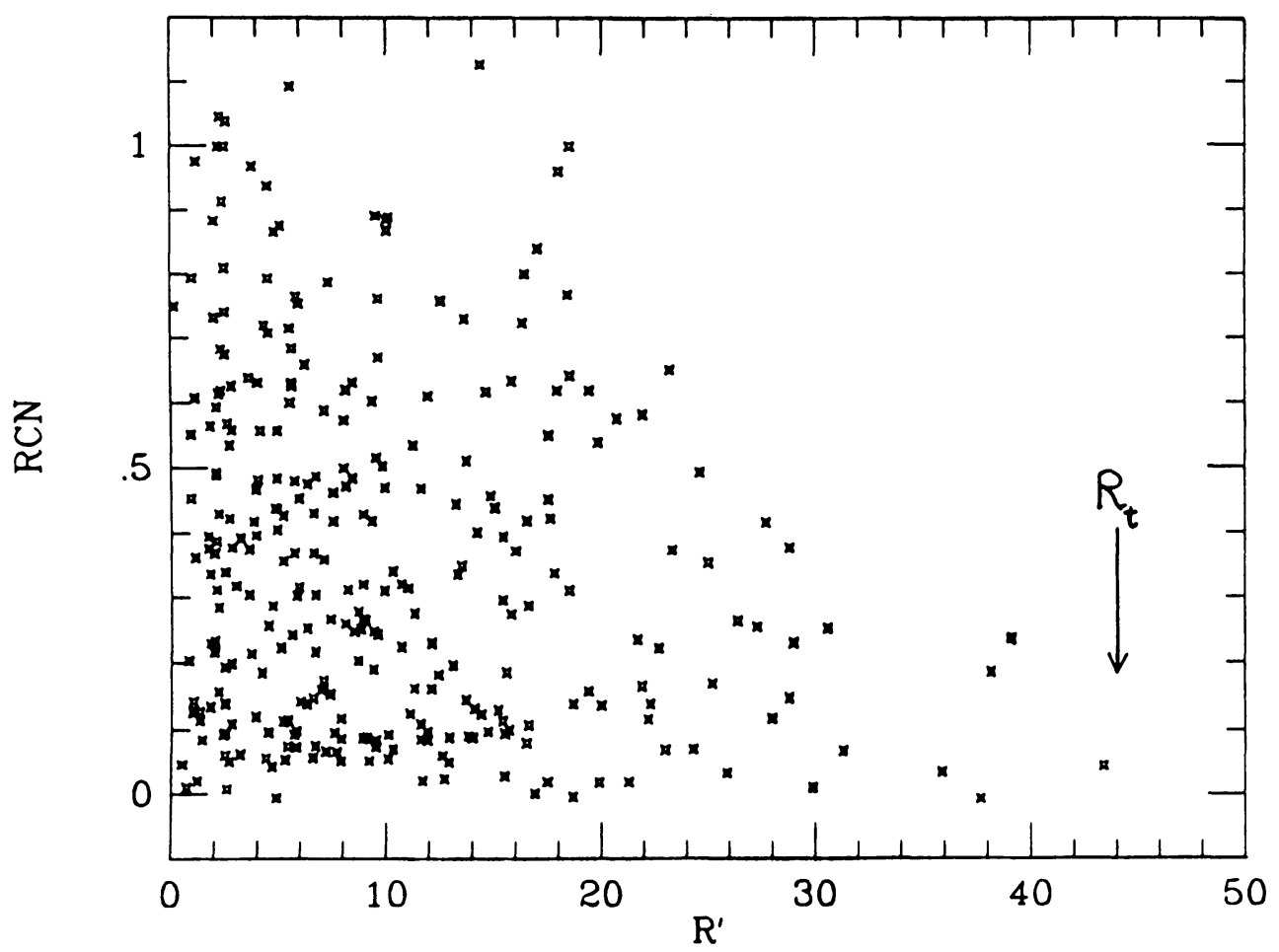

Figure 4. Radial distribution of the $\mathrm{CN}$ strength $\mathrm{RCN}$ (RCN increases with increasing $\mathrm{CN}$ strength) for stars in Omega Cen. The $\mathrm{CN}$ gradient is obvious. The arrow marked $\mathrm{R}_{t}$ is at the tidal radius.

their velocity dispersion becomes low. The stars near this maximum radius are the most energetic stars of their $\mathrm{CN}$ class, seen now near their maximum distance from the cluster center and therefore with low velocities. Similarly, the velocity dispersion of the intermediate and weak CN subsamples becomes low towards the maximum radius at which such stars are found. So, in Omega Cen, the kinematics and the radial distribution of $\mathrm{CN}$ give us a consistent picture of the chemical gradient, in whirh the CN-strong stars are the ones most bound to the cluster center.

Omega Cen is a relatively flattened cluster. Analysis of the stellar velocities shows that the cluster is rotating. The position angle of the kinematic major axis agrees very well with that for the major axis determined from the star count distribution. In the $(V / \sigma)-\varepsilon$ plane ( $V$ is the maximum rotational velocity, $\sigma$ is the central velocity dispersion, and $\varepsilon=1-b / a$, where $\mathrm{a}$ and $\mathrm{b}$ are the apparent 


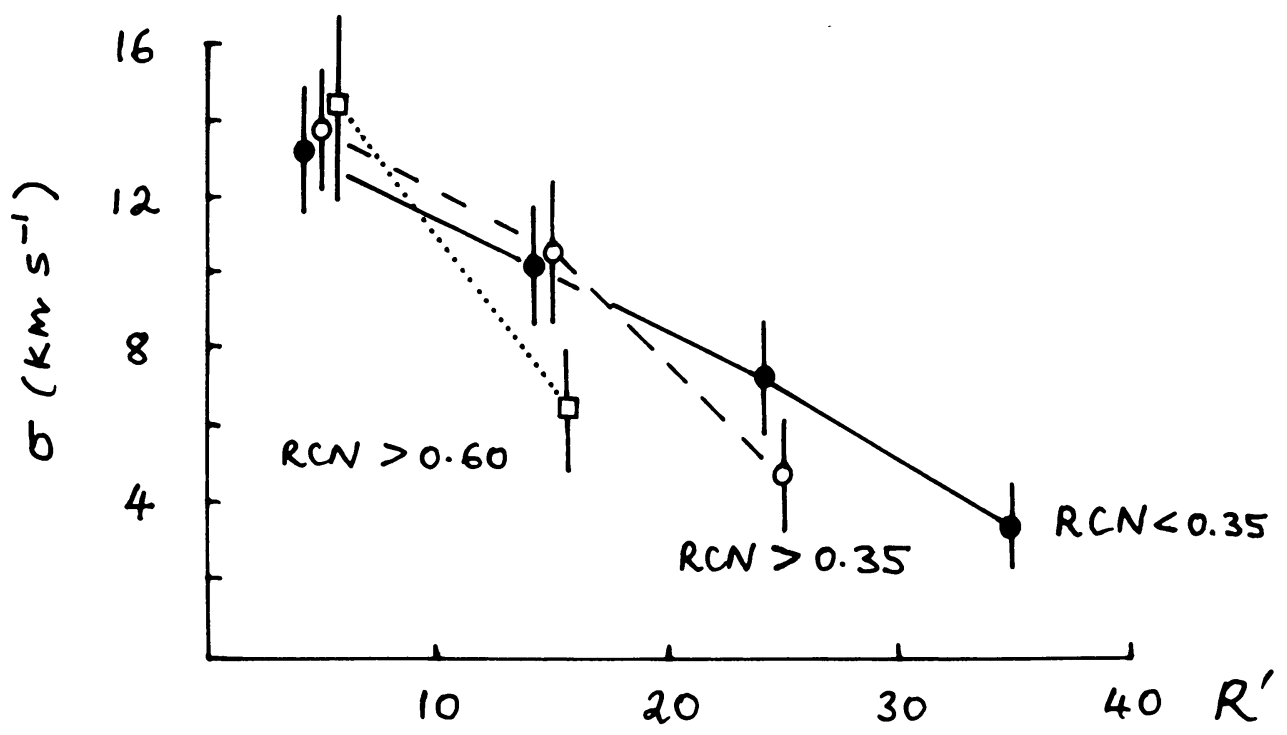

Figure 5. The radial variation of stellar velocity dispersion in Omega Cen, for the three CN-strength subsamples shown by the labels. The velocity dispersion decreases most rapidly with radius for the CN-strongest subsample, which is the most concentrated to the cluster center.

major and minor axes of the star count distribution at the count level corresponding to the maximum observed flattening), the cluster lies close to the oblate isotropic line (see seitzer et al, 1984) so it seems very likely that Omega Cen is flattened by its rotation.

It seemed worth comparing the rotation for the $\mathrm{CN}$-weak ( $\mathrm{RCN}<0.35$ ) and $\mathrm{CN}$-strong ( $\mathrm{RCN}>0.35)$ stars in Omega Cen. From our sample, it appears that the subsystem of $\mathrm{CN}$-weak stars is rotating significantly more rapidly than the $\mathrm{CN}$-strong subsystem. This result is somewhat unexpected, and more stars with good velocities and $\mathrm{CN}$ measures are needed in the inner parts of the cluster to check its validity. We know that, in galaxies, the dependence of rotation on chemical abundance is the other way around: the metal rich component is the more dissipated and rapidly rotating. However Omega Cen is unique among the clusters with its heavy element inhomogeneity. If this inhomogeneity results from the coalescence of two or more fragments of different abundance, then the chemical gradient and the difference in rotation of the CN-weak and $\mathrm{CN}$-strong components probably reflects the different dynamical properties of these fragments before coalescence. On the other hand, if self pollution produces the inhomogeneity and the chemical gradient, then the rapid rotation of the $\mathrm{CN}$-weak component becomes an interesting part of the formation picture. We recall again that the relaxation time in Omega Cen is very long, so the dynamical properties of the cluster are probably much as they were immediately after the time of formation. 


\section{REFERENCES}

Butler, D., Dickens, R.J. and Epps, E. 1978. Ap.J. 225, 148.

Chun, M.S. 1976. Australian National University Thesis.

Chun, M.S. and Freeman, K.C. 1979. Ap.J. 227, 93.

d'Antona, F., Gratton, R. and Chieffi, A. 1983. Mem.Soc.Astron. Ital., 54, 173.

Freeman, K.C. 1978. Nato Advanced Study Institute: Globular Clusters ed. D. Hanes and B. Madore, p103. Cambridge University Press.

Freeman, K.C. and Norris, J.E. 1981. Annual Reviews of Astr. and Astrophys. 19, 319.

Gascoigne, S.C.B. and Burr, E.J. 1956. Mon.Not.Roy.Astron.Soc. 116, 570.

Martin, W.Ch.1937. Leiden University Thesis.

Norris, J.E. 1981. Ap.J. 248, 177.

Norris, J.E. and Freeman, K.C. 1979. Ap.J. 230, L179.

Norris, J.E. and Smith, G.H. 1981. Astrophysical Parameters for

Globular Clusters, ed. A.G. Davis Philip and D.S. Hayes, plog.

L. Davis Press, Schenectady.

Searle, L. and Zinn, R. 1978. Ap.J. 225, 357.

Seitzer, P., Freeman, K.C. and Norris, J.E. 1984. To be published.

Smith, H.A. 1979. A.J. 84, 176.

Smith, H.A. 1981. A.J. 86, 538 


\section{DISCUSSION}

KING: Most of these abundance gradients are mystifying, but one result is quite astounding. Your high-CN stars have just as great a velocity dispersion at the center, but both their density distribution and their velocity dispersion drop off much faster. Is there any rational model that will fit this?

FREEMAN: Yes. If we write your lowered Maxwellian as $f(E) \approx\left(e^{-E / \sigma^{2}}-e^{-E_{O} / \sigma^{2}}\right)$, then the $\mathrm{CN}-$ rich stars would need a somewhat higher value of the scale dispersion $\sigma$ and a somewhat more bound value of the cutoff energy $E_{o}$ to produce the observed density and velocity dispersion behavior.

LARSON: Does any of the evidence rule out the possibility that $\omega$ Cen is just a superposition of two clusters, a metal-rich one and a metal-poor one, that merged, and that all of the observed radial trends just represent varying relative proportions of the two populations of stars?

FREEMAN: The CN-distribution in $\omega$ Cen is not bimodal, as it is in many other clusters. It is a broad and fairly continuous distribution, which would argue against your suggestion.

HANES: How strictly correlated, star-by-star, are the $\mathrm{CN}$ and $\mathrm{Ca}$ variations? Obviously, if the Ca bimodality is primordial, its correlation with $\mathrm{CN}$ tells you something about the origin of variations in the latter.

FREEMAN: The correlation is indeed fairly well defined, which does support a primordial origin for the chemical inhomogeneities, as you suggest.

OSTRIKER: A variety of calculations has shown that angular momentum diffuses outwards in related systems. Thus the metal rich components would be expected to "spin down" somewhat. Can this effect quantitatively account for a lower rotational velocity of the CN rich component in $\omega$ Cen?

FREEMAN: It seems unlikely, unfortunately. In $w$ Cen, the relaxation time is close to the Hubble time, even in the center of the cluster.

LACHIEZE-REY: A correlation also exists between color gradient and rotation in elliptical galaxies. As a result of a multicolor CCD photometry of elliptical galaxies we found that color (B-R) gradients exist which cannot be reduced to metallicity gradients and that they correlate well to the quantities $(v / \sigma)$ or $(v / \sigma) *$. 